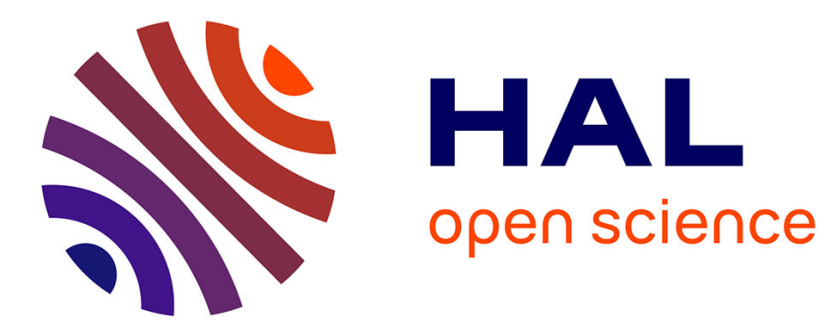

\title{
ETUDE DU BRUIT ENGENDRÉ PAR L'IMPACT D'UN JET SUPERSONIQUE CHAUD SUR UN OBSTACLE
}

B. Jacquet, D. Gély

\section{- To cite this version:}

B. Jacquet, D. Gély. ETUDE DU BRUIT ENGENDRÉ PAR L'IMPACT D'UN JET SUPERSONIQUE CHAUD SUR UN OBSTACLE. Journal de Physique IV Proceedings, 1992, 02 (C1), pp.C1569-C1-572. 10.1051/jp4:19921123 . jpa-00251079

\section{HAL Id: jpa-00251079 https://hal.science/jpa-00251079}

Submitted on 1 Jan 1992

HAL is a multi-disciplinary open access archive for the deposit and dissemination of scientific research documents, whether they are published or not. The documents may come from teaching and research institutions in France or abroad, or from public or private research centers.
L'archive ouverte pluridisciplinaire HAL, est destinée au dépôt et à la diffusion de documents scientifiques de niveau recherche, publiés ou non, émanant des établissements d'enseignement et de recherche français ou étrangers, des laboratoires publics ou privés. 


\title{
ETUDE DU BRUIT ENGENDRE PAR LIMPACT D'UN JET SUPERSONIQUE CHAUD SUR UN OBSTACLE 1
}

\author{
B. JACQUET et D. GELY \\ Office National d'Etudes et de Recherches Aérospatiales, BP. 72, F-92322 Châtillon cedex, France
}

RESUME

Une étude expérimentale du bruit engendré par l'interaction d'un jet supersonique chaud avec un obstacle est présentée. La caractérisation des sources de bruit a été effectuée par des mesures de directivités en champ lointain, des mesures de puissance acoustique rayonnée et des localisations de sources par réseau de capteurs. L'introduction d'un obstacle dans un jet entraîne une élévation des niveaux sonores dans la partie de l'espace en amont de l'obstacle. La localisation de sources a montré que les sources de bruit prépondérantes sont situées dans la zone d'interaction. Cependant, l'apparition de ces sources ne se traduit pas par une augmentation importante de la puissance rayonnée.

\section{ABSTRACT}

This document presents experimental works on supersonic hot jet impingement noise which have been done in an anechoic wind tunnel. Far field noise directivity measurements were done for a supersonic jet impingement on an axisymmetric obstacle. Source localizations with focused microphone arrays were realized simultaneously. Introduction of a board in a jet increases the sound pressure level upstream of the board. The localizations with focused microphone arrays show that the predominant sources of noise are located near the impingement region. However, this apparition of sound sources does not amount to a large increase of the total sound power level.

\section{INTRODUCTION}

Dans le cadre de la modélisation de l'ambiance acoustique des lanceurs Ariane, l'ONERA a réalisé une étude expérimentale du champ sonore rayonné par l'impact d'un jet supersonique sur un obstacle à symétrie de révolution. La caractérisation de ce champ a été effectuée par des mesures de puissance acoustique émise et par des mesures de directivité en champ lointain. Par ailleurs, l'utilisation d'un réseau linéaire de capteurs a permis la localisation des sources de bruit liées à l'interaction du jet avec l'obstacle.

\section{DESCRIPTION DU MONTAGE EXPERIMENTAL}

La campagne d'essais s'est déroulée dans la soufflerie anéchoïque CEPRA 19 au Centre d'Essais des Propulseurs (CEPr). Le jet est issu d'une tuyère convergente de diamètre $D=60 \mathrm{~mm}$ (fig. 1). Les conditions génératrices sont un taux de détente de 3,4 et une température génératrice de $1050 \mathrm{~K}$. Le jet a donc un nombre de Mach local de 1,43 et une vitesse de décharge de $780 \mathrm{~ms}^{-1}$.

Le dispositif servant d'obstacle est constitué d'une plaque circulaire de $1 \mathrm{~m}$ de diamètre (fig. 1). Il est possible d'étudier l'influence de la forme de l'obstacle en modifiant la partie centrale de ce dispositif. Les formes d'obstacles

'Cette étude a reçu le soutien du CNES 
utilisées au cours des expériences sont toutes à symétrie de révolution (fig. 1) :

- $\operatorname{plan}(\mathrm{P})$,

- plan avec un alésage de diamètre D (PT1D),

- plan avec un alésage de diamètre 20 (PT2D),

- plan avec un cóle central pointé vers l'amont dont la hauteur est de 3D (C3D).

Les mesures de directivité en champ lointain sont réalisées sur un arc de cercle de $6 \mathrm{~m}$ de rayon centré sur la sortie tuyère (fig. 1). Cette analyse spatiale du champ sonore est réalisée par pas de $10^{\circ}$ pour des angles compris entre $30^{\circ}$ et $150^{\circ}\left(0^{\circ}\right.$ sur l'axe du jet, vers l'aval).

Un réseau linéaire de capteurs est utilisé pour localiser les sources acoustiques. La position de l'antenne acoustique est donnée sur la figure 1. Les images acoustiques sont obtenues par la méthode de formation de voie [1] pour 128 raies spectrales comprises dans la gamme de fréquences $200 \mathrm{~Hz}-15 \mathrm{kHz}$.

Un montage ombroscopique a permis de visualiser les interactions entre un obstacle et un jet supersonique froid $(\mathrm{M}=1,43)$.
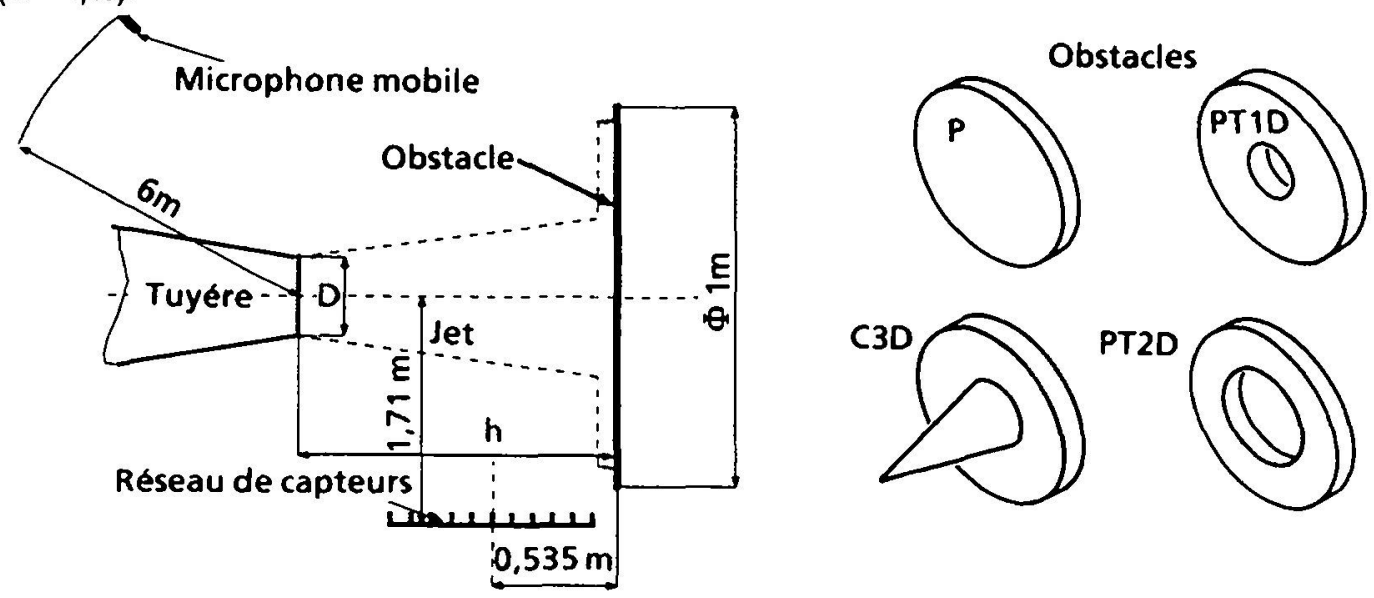

Figure 1 Montage expérimental

\section{RESULTATS EXPERIMENTAUX}

\subsection{Visualisations ombroscopiques}

La figure 2 présente un exemple de visualisation ombroscopique réalisée lors d'une interaction entre un jet supersonique froid et un obstacle plan, la distance h entre le plan de sortie tuyère et l'obstacle étant de $6 \mathrm{D}$. Une onde de choc apparaît en amont de l'obstacle dans toutes les configurations d'interaction. Sa forme est directement liée à celle de l'obstacle et son intensité décroît lorsque la distance entre l'obstacle et le plan de sortie tuyère augmente.

La comparaison des visualisations d'interaction avec celle du jet libre (fig. 3) montre que l'introduction d'un obstacle dans un écoulement supersonique n'engendre des modifications du cóne potentiel que dans une petite zone de dimension caractéristique D située juste en amont de l'obstacle.

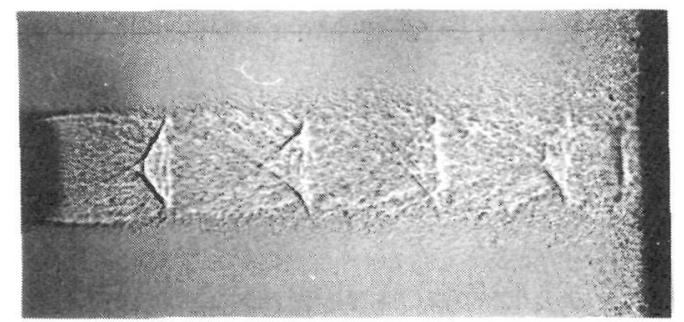

Figure 2 Visualisation d'une interaction avec l'obstacle $\mathrm{P}$ placé à la distance $\mathrm{h}=6 \mathrm{D}$.

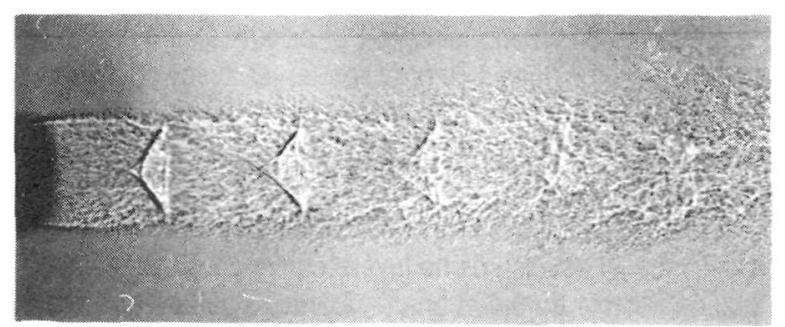

Figure 3 Visualisation du jet libre 


\subsection{Localisation des sources}

La figure 4 présente la position des sources de bruit lors d'une interaction entre un jet chaud supersonique et un obstacle de forme plane (P) placé à la distance $h=15 \mathrm{D}$. Il apparaît clairement que, pour toutes les fréquences, les sources de bruit prépondérantes sont situées dans la zone d'interaction. La comparaison avec le cas du jet libre (fig. 5) montre que les sources de bruit d'interaction masquent totalement celles du jet libre.

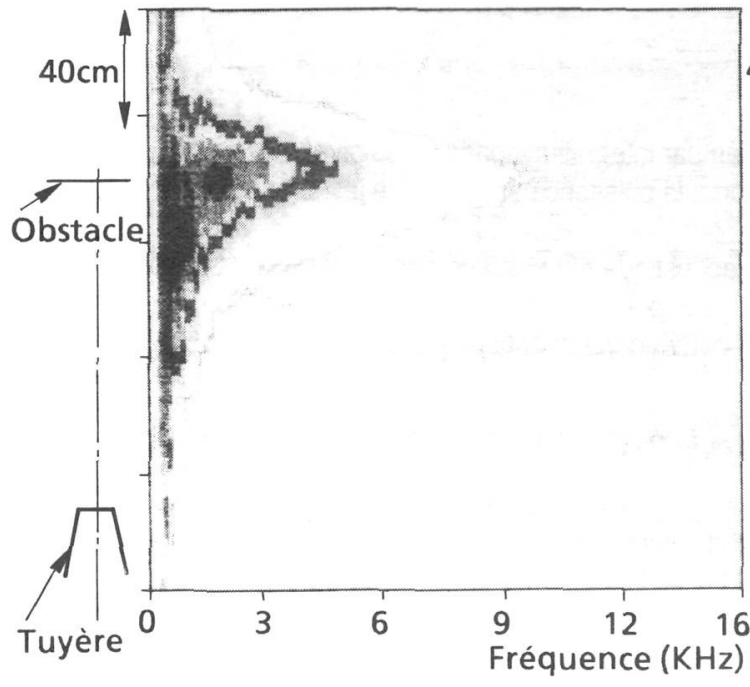

Figure 4 Position des sources lors d'une interaction avec l'obstacle plan placé à la distance $h=15 \mathrm{D}$.

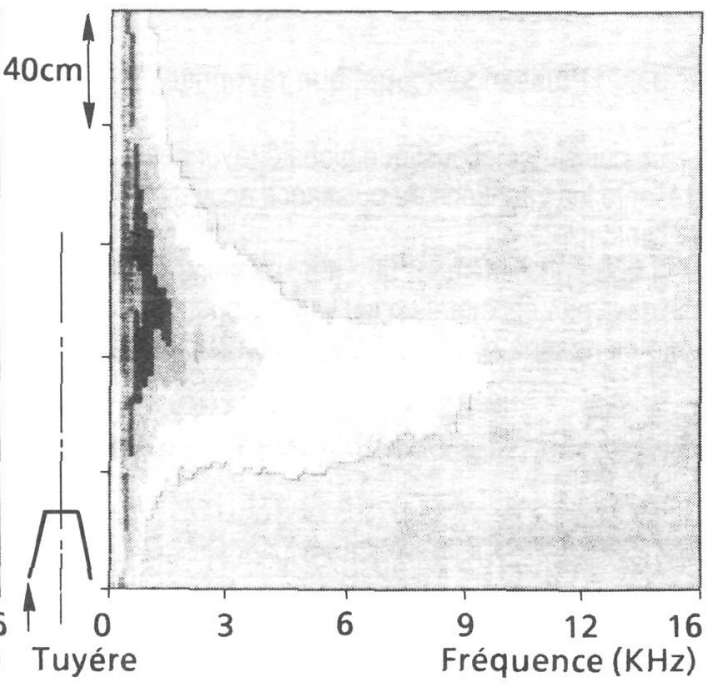

Figure 5 Position des sources dans le jet libre.

\subsection{Directivites}

La figure 6 présente les directivités mesurées lors d'interactions avec des obstacles de différentes formes. Sur les courbes présentées, la distance h est de 9 D. La directivité du jet libre est aussi indiquée sur cette planche.

La directivité du champ sonore engendré par l'impact d'un jet sur un obstacle de grande dimension se caractérise par un minimum de niveau dans la partie de l'espace situé dans le plan de l'obstacle et par une augmentation sensible du niveau sonore, de l'ordre de $10 \mathrm{~dB}$, en amont de l'obstacle. Le maximum de directivité est obtenu pour une position angulaire comprise entre $120^{\circ}$ et $140^{\circ}$ pour toutes les configurations d'interaction étudiées.

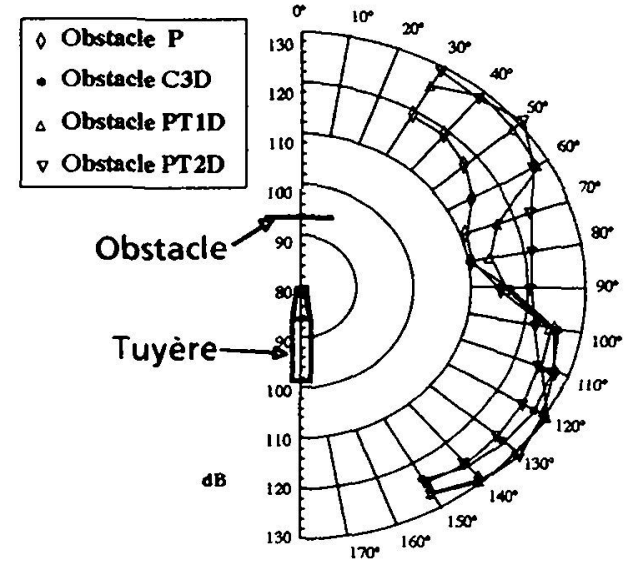

Figure 6 Directivités mesurées pour différents obstacles placés à la distance $h=9 \mathrm{D}$.

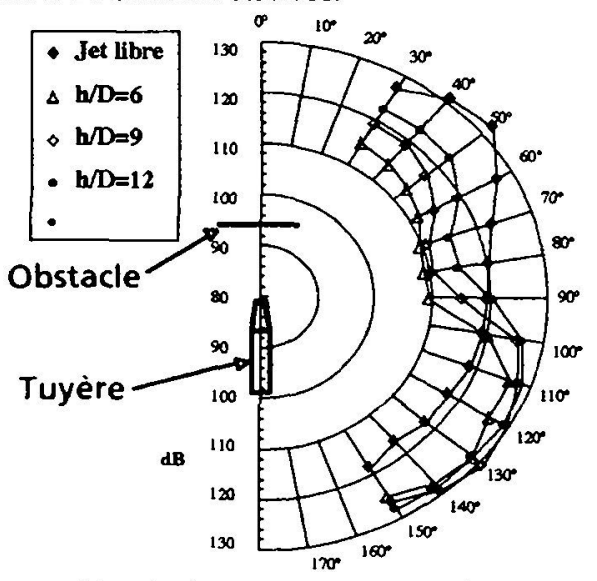

Figure 7 Directivités mesurées pour différents rapports h/D lors d'interactions avec l'obstacle plan. 
L'analyse comparative des directivités montre que la forme centrale de l'obstacle influence peu les caractéristiques spatiales du champ sonore en amont de l'obstacle. De plus, cette influence s'atténue lorsque la distance d'interaction augmente.

La figure 7 présente les directivités mesurées lors d'interactions avec un obstacle plan situé à différentes distances du plan de sortie tuyère. Pour des distances d'interaction comprises entre $6 \mathrm{D}$ et $15 \mathrm{D}$, l'allure générale de la directivité est globalement indépendante de la distance d'interaction. Le maximum de niveau en amont de l'obstacle est obtenu pour une distance $h \approx 12 \mathrm{D}$.

\subsection{Puissance acoustique rayonnée}

La puissance acoustique globale rayonnée a été calculée par intégration spatiale des niveaux sonores. La figure 8 présente les variations de puissance acoustique, par rapport à la puissance émise par le jet libre, dues à l'introduction de l'abstacle.

II apparaît nettement que les variations de puissance acoustique engendrées par la présence de l'obstacle sont faibles. Cette constatation est valable pour tous les obstacles et pour toutes les distances d'interaction étudiés lors de cette campagne d'essais. La variation la plus significative obtenue est inférieure à $3 \mathrm{~dB}$.

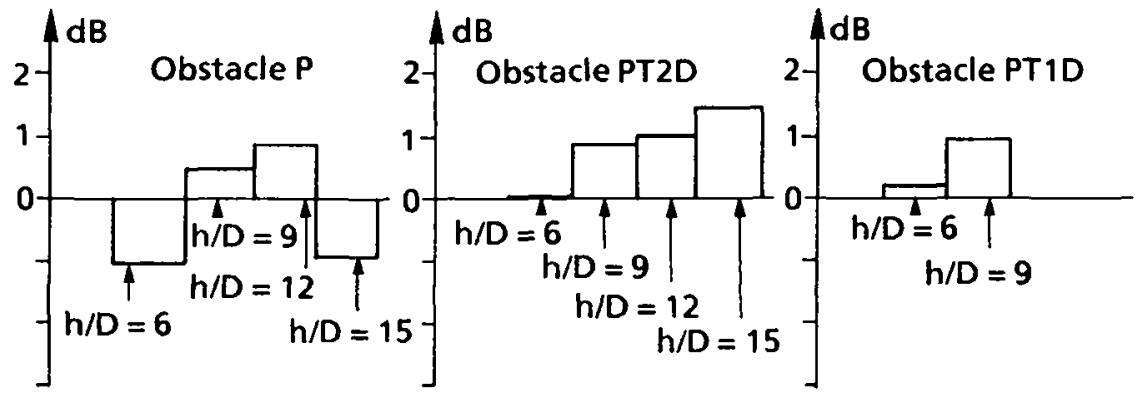

Figure 8 Variations de la puissance acoustique rayonnée engendrées par l'introduction de l'obstacle, par rapport au jet libre.

\section{CONCLUSION}

L'étude acoustique réalisée a permis d'établir plusieurs caractéristiques du champ sonore dû à l'interaction entre un jet chaud supersonique et un obstacle de grande dimension. L'introduction d'un obstacle dans un jet entraîne une élévation des niveaux sonores dans la partie de l'espace en amont de l'obstacle. La localisation de sources a montré que les sources de bruit prépondérantes étaient situées dans la zone d'interaction. Cependant, l'apparition de ces sources ne se traduit pas par une augmentation importante de la puissance totale rayonnée, contrairement au cas des jets subsoniques. Cette constatation est limitée à des jets faiblement supersoniques. D'autres expériences avec des jets de plus fortes vitesses sont donc nécessaires pour s'assurer de la généralité de cette propriété du bruit d'interaction.

\section{REFERENCE}

[1] G. Elias, C. Malarmey, Revue d'Acoustique Hors Série, 11ème Congrès International d'Acoustique, Paris, pp. 163-166, 1983. 